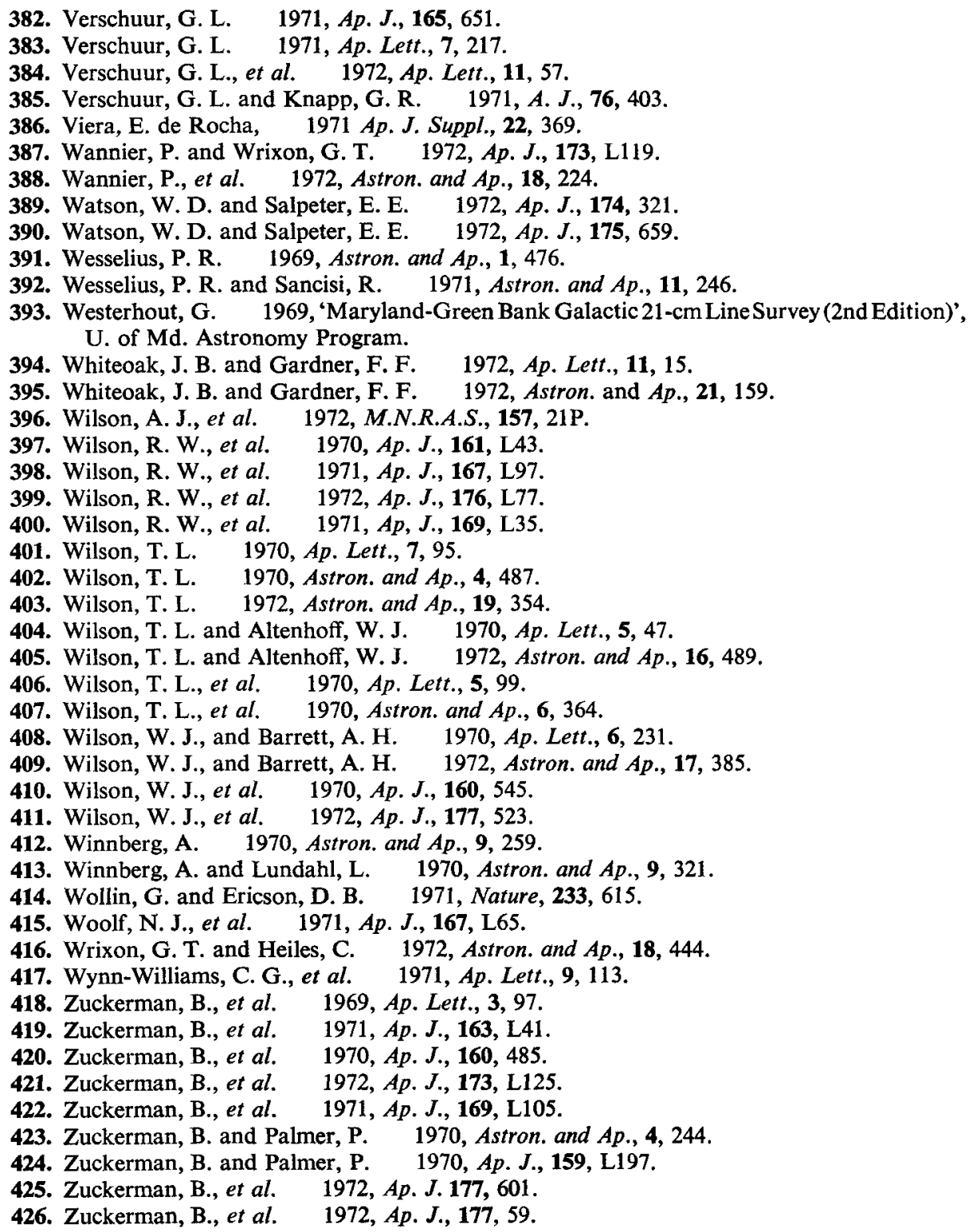

\title{
D. EXTRAGALACTIC RADIO ASTRONOMY 1969-1972
}

\section{H. van der Laan and G. K. Miley}

Radio astronomy shows increasing astrophysical diversity and the extragalactic branch ranges from spiral structure through relativistic astrophysics of nuclei of galaxies and quasars to observational cosmology. A bibliographic listing of about 700 papers under almost forty headings and subheadings has been prepared which systematically covers the subjects within our terms of reference. We list all relevant articles published in the journals listed in Section B(i), covering the period from July 1969 to November 1972. Comprehensive accounts of the subject may be found in the 
1971 proceedings of the Vatican Semaine d'Etude and the Uppsala IAU Symposium No. 44. These are listed under 'Reviews' and the individual articles have not been included separately in any other category. Each publication is designated by a minimal, abbreviated reference.

Some features of the work covered in this report are the rapid growth of data for radio sources and the promising development of radio investigations of optically selected objects. Many surveys have added to the primary reservoir of catalogued sources which merit further study. These surveys now span a large frequency range and the intercomparisons have clarified the spectral relations of source counts and the frequency dependence of the radio luminosity function. Very deep surveys now in progress will hopefully enable selection effects to be distinguished from cosmologically relevant features.

Improved positional accuracy, provided especially by phase stable interferometry, has led to a large harvest of optical identifications and subsequent spectroscopic and photometric work reported in other commissions.

The last three years have seen the increasing use of the Earth rotation synthesis technique to derive high resolution brightness maps of extragalactic sources. The detailed brightness distributions of many sources was explained with some success by invoking ram pressure confinement by an extragalactic medium. Circular polarization was detected in several sources and a few one and two dimensional linear polarization maps were obtained. It is hoped that the wealth of high resolution polarization data which will shortly become available will help to restrict the range of magnetohydrodynamic confinement models. Also, since both angular size and linear polarization have been shown to be well correlated with redshift, a study of the polarization distributions of high redshift objects may well impose useful constraints on possible cosmological models.

The use of very long baseline interferometers has become more widespread and one of the most interesting 'VLBI' results has been the observation of apparent expansion in a number of compact radio sources. For some quasars these data might imply highly relativistic expansion ('superlight velocities') if the quasar redshifts are cosmological in origin. Although their interpretation is still uncertain, it appears probable that the expansions are not real but, merely an effect produced by intensity variations between spatially separated regions within the sources.

Increasingly radio telescopes are turned, not to sources first listed in radio catalogues, but to nearby classical stellar systems and moderately distant optically conspicuous galaxies. In the period reported here a promising start was made with high resolution studies of neutral hydrogen distribution and kinematics in nearby galaxies. Combined with interferometric continuum studies these investigations have begun a new phase of spiral galaxy research. The advent of high resolution aperture synthesis telescopes and advances in electronic sensitivity will no doubt lead to many complementary and joint programmes of astronomers working in different spectral domains. For the investigation of radio galaxies and quasars this has been an impressively fruitful cooperation. It is to be expected that such combined efforts for nearer objects, much more amenable to detailed exploration, will soon result in exciting progress.

\section{A. Contents of Bibliography}

I. Surveys

II. Flux Density Measurements

(i) Optically selected sources: Detections and Measurements

(ii) Other flux density measurements

(iii) Flux scales and absolute measurements

III. Position Measurements

(i) Better than $\sim 1^{\prime \prime}$

(ii) Better than $\sim 5^{\prime \prime}$

(iii) Less accurate

(iv) Position comparisons 
IV. Brightness Distribution Measurements

(i) Pencil beam

(ii) Interferometric: Baselines $<3 \mathrm{~km}$

(iii) Interferometric: Baselines $>3 \mathrm{~km}$

(iv) Occultation

(v) Scintillation

V. Polarization Measurements

(i) Integrated linear polarizations

(ii) Linear polarization distributions

(iii) Circular polarization

VI. Neutral Hydrogen Measurements

(i) Emission/Pencil beam

(ii) Emission/Interferometric

(iii) Redshifted absorption

VII. Microwave Background Measurements

VIII. Miscellaneous Observations

IX. Optical Identifications

X. Interpretation of Spectral Data

XI. Interpretation of Variability Data/Compact Sources

XII. Interpretation of Structure Data

XIII. Interpretation of Polarization Data

XIV. Redshift Dependent Properties of Sources

XV. Miscellaneous Correlations

XVI. Interpretation of Microwave Background Data

XVII. Theory of Radio Sources

(i) Radiation Theory

(ii) Effects of Compton Scattering

(iii) Source Models

(iv) Magnetohydrodynamics/Source confinement

XVIII. Source Counts and Related Topics

(i) Data

(ii) Interpretation and luminosity functions

XIX. Miscellaneous Radio-Cosmology

XX. Review Papers etc.

(i) Compendia of data

(ii) Reviews

(i)

B. Abbreviations

$A A \quad$ Astronomy and Astrophysics

AASup Astronomy and Astrophysics Supplements

$A d v A A$ Advances in Astronomy and Astrophysics

$A J \quad$ Astronomical Journal

$A J P \quad$ Australian Journal of Physics

AJPSup Australian Journal of Physics Astrophysical Supplements

AnRev Annual Reviews of Astronomy and Astrophysics

Aph Astrophysica

$A p S p S c$ Astrophysics and Space Science

ApJ Astrophysical Journal 


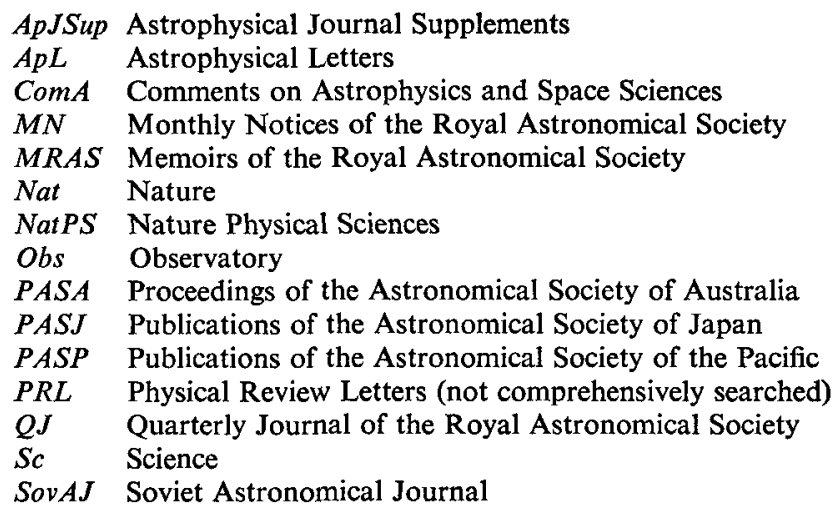

(ii) Column headings

frq, freqs frequency or frequency range of observations in gigahertz

flx min minimum flux density in survey/in units of $10^{-26} \mathrm{~W} \mathrm{~Hz}^{-1} \mathrm{~m}^{-2}$

N No. of sources dealt with in article

(iii) Number of sources dealt with in article; $N$

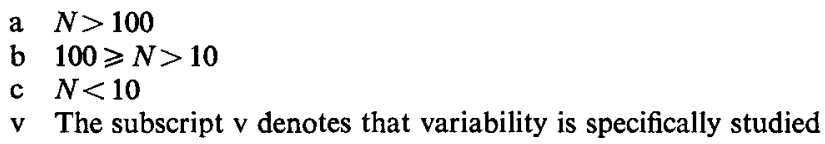

(iv) Notes and comment

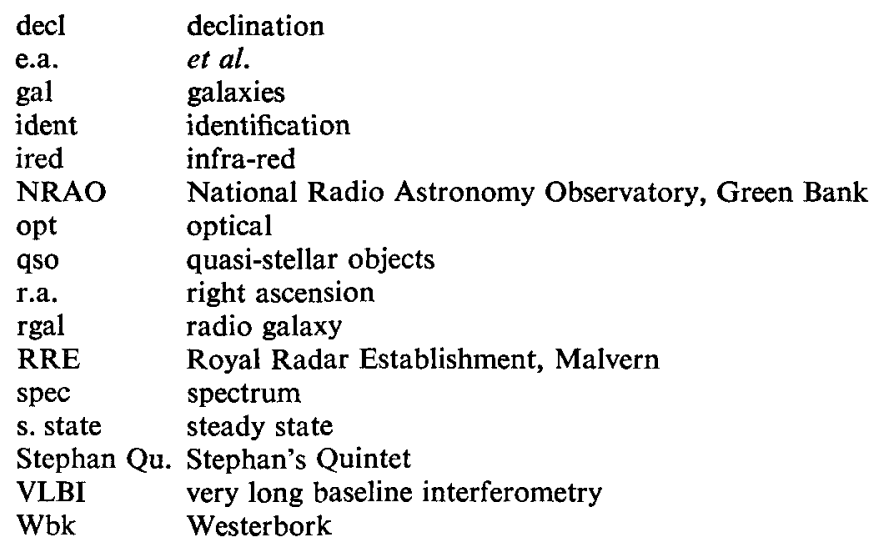

I. SURVEYS

\begin{tabular}{|c|c|c|c|c|c|c|}
\hline frq & $\begin{array}{l}\mathrm{flx} \\
\min \end{array}$ & $\mathbf{N}$ & frq & $\begin{array}{l}\mathrm{flx} \\
\min \end{array}$ & $\mathbf{N}$ & \\
\hline .18 & 2.5 & a Caswell, Crowther $69 M N 145,181$ & 1.4 & .2 & a 1 & Ehman e.a. $70 A J 75,351$ \\
\hline .41 & .01 & a Pooley $69 M N 144,101$ & 1.4 & .2 & a & Brundage e.a. $71 A J 76,777$ \\
\hline 41 & .07 & a Windram, Kenderdine $69 M N 146,265$ & 1.4 & .09 & & Maslowski 71 AA14, 215 \\
\hline .41 & .2 & a Davies $70 P A S A 1,340$ & 1.4 & .09 & & Maslowski 72AA16, 197 \\
\hline .41 & .2 & a Colla e.a. $70 A A \operatorname{Sup} 1,281$ & 1.4 & .2 & a & Willson $72 M N 156,7$ \\
\hline .41 & .02 & a Willson $70 M N 151,1$ & 2.7 & .1 & a & Wall e.a. 71 AJPSup 19 \\
\hline .41 & .6 & a Willson $72 M N 156,7$ & 2.7 & .3 & & Shimmins 71 AJPSup 21 \\
\hline .41 & .2 & a Colla e.a. $72 A A S u p 7,1$ & 5.0 & .07 & a & Davis $71 A J 76,980$ \\
\hline .61 & .8 & a Wendker e.a. $70 A J 75,148$ & 5.0 & .09 & a & Pauliny-Toth e.a. $72 A J 77,265$ \\
\hline .61 & .8 & a Dickel e.a. $71 A J 76,294$ & & & & \\
\hline
\end{tabular}




\section{FLUX DENSITY MEASUREMENTS}

(i) Optically selected sources: Detections and Measurements

\begin{tabular}{|c|c|c|}
\hline Object & Freqs & $N$ \\
\hline Gal & .41 & b Longair e.a. $70 A p L 7,23$ \\
\hline Gal & .41 & a Cameron $71 M N 152,403$ \\
\hline Gal & 1.4 & $\begin{array}{l}\text { b van der Kruit } 71 A A 15 \text {, } \\
\quad 110\end{array}$ \\
\hline Gal & 2.6 & $\begin{array}{l}\text { a Rogstad, Ekers } A p J 157 \text {, } \\
481\end{array}$ \\
\hline Gal & 2.7 & b Kazés e.a. $70 A p L 6,193$ \\
\hline Gal & $2.7,15$ & a Heeschen $70 A J 75,523$ \\
\hline Gal & 5.0 & a Whiteoak $70 A p L 5,29$ \\
\hline Gal & 8.5 & $\begin{array}{l}\text { c Kuril'chik e.a. } 70 \text { SovAJ } \\
13,881\end{array}$ \\
\hline Maffei1 & & Oort 71 Nat 230, 103 \\
\hline Maffei2 & $.18,1.4$ & Caswell 71 Nat 231, 35 \\
\hline
\end{tabular}

(ii) Other fux density measurements

\begin{tabular}{|c|c|}
\hline Freqs & $N$ \\
\hline .01 & $c_{v}$ \\
\hline $\begin{array}{l}.02 \\
.04,5\end{array}$ & $\begin{array}{l}3 \mathrm{C} 84 v \\
a(3 C)\end{array}$ \\
\hline $.32, .61$ & $\mathrm{~b}$ \\
\hline $.01, .03$ & $b$ \\
\hline $.41,1.4$ & b \\
\hline $.41,5.0$ & P1514-24 \\
\hline .41 & b \\
\hline .41 & $a(4 C)$ \\
\hline $.61,13.5$ & $\mathbf{b}$ \\
\hline .41 & $a(4 C)$ \\
\hline .41 & $a(4 C)$ \\
\hline .43 & $\mathrm{a}$ \\
\hline $.61,11$ & b(Ohio) \\
\hline $.61,11$ & b(Ohio) \\
\hline $0.61,85$ & $\mathrm{c}$ \\
\hline .75 & $\mathrm{~b}$ \\
\hline $.75,11$ & $c_{v}$ \\
\hline 1.4 & $\mathrm{a}$ \\
\hline 1.4 & $\mathrm{~b}(5 \mathrm{C} 1)$ \\
\hline 1.4 & $\mathrm{~b}(5 \mathrm{C} 1 / 2)$ \\
\hline
\end{tabular}

Bridle, Caswell 70Nat 225, 356

Roger 69ApL4, 139

Kellermann e.a. $69 \mathrm{ApJ}$

157, 1

Jauncey e.a. $70 A p J$

162, L31

Braude e.a. $70 A p L 5$, 129

Hunstead, Jauncey

$70 M N 149,91$

Hunstead 71 Nat 233, 401

Jauncey $72 A J 77,345$

Fanti e.a. $69 A A 2$,

477

Wills e.a. $71 A p J 169$,

L87

Munro 71 AJP24, 263

Munro 72AJPSup22

Backer e.a. $70 A J 75$,

529

Kraus, Andrew

70ApJ159, L41

Kraus, Andrew

70ApJ159, L45

Andrew e.a.

71PASP83, 87

Rzhiga, Frunova

69SovAJ13, 28

Ross 70Nat 226, 431

Fomalont, Moffet

71AJ76, 5

Maslowski $71 \boldsymbol{A A 1 4}$,

215

Maslowski $72 A A 16$, 197

\begin{tabular}{|c|c|c|}
\hline Object & Freqs & $N$ \\
\hline Maffei2 & $3.2,11$ & Bell e.a. $70 A p J 161, \mathrm{L13}$ \\
\hline Maffei2 & $3.2,11$ & $\begin{array}{l}\text { Bell, Seaquist } 72 A p J 173 \text {, } \\
257\end{array}$ \\
\hline Maffei2 & $.61,2.7$ & $\begin{array}{l}\text { Webber, Willis } 71 \text { Nat } 231 \text {, } \\
36 \text {. }\end{array}$ \\
\hline NGC604 & 1.4 & Wright $71 A p L 7,209$ \\
\hline NGC5128 & $2.7,8.1$ & Wade e.a. $71 A p J 170$, L 11 \\
\hline QSO & .32 & $\begin{array}{l}\text { b Lang, Terzian } 69 A p J 158 \text {, } \\
\text { L11 } \\
\text { Grueff } 70 A p J 160, \text { LA1 }\end{array}$ \\
\hline QSO & $2.7,8.1$ & $\begin{array}{l}\text { b Wardle, Miley } 71 A p J 164 \\
\text { L119 }\end{array}$ \\
\hline QSO & 31,86 & b Conklin 70 Nat 227, 1119 \\
\hline
\end{tabular}

Freqs $\quad N$

1.4 b(5C3) van der Kruit,

$1.4 \quad$ a

$1.4 \quad \mathrm{BLLac}_{\mathrm{v}}$

$2.3 \quad c_{v}(\mathrm{VLBI})$

atgert $72 A p L 11,181$

Bridlee.a. 72AJ77,405

Gower 69Nat 224, 569

69Nat 224, 1094

Nicolson

71 NatPS233, 155

2.3 P2204-24 Tritton, Nicolson

72ApL11, 187

$2.7 \quad \mathrm{a}(4 \mathrm{C})$

$2.7 \quad b$

$2.7,5.0 \quad a$

$2.7 \quad b_{\mathbf{v}}$

$2.7 \quad b_{v}$

2.7 bv

$2.7 \quad b$

$2.7,5.0 \quad \mathrm{c}$

Wall 71AJPSup20

Ames 70AJ75, 71

Witzel e.a. $71 A A 11$, 171

Witzel, Veron

71ApL7, 225

Wills 71 ApJ169, 221

Grahl, Grewing

69ApLA, 107

Browne, McEwan

72NatPS239, 101

Fanaroff, Blake

72MN157, 41

$2.7 \quad \mathrm{c}$ (Ohio) Kraus, Andrew

71AJ76, 103

2.7,5 a(weak) Pauliny-Toth, Kellermann $72 A J 77,560$

3.2, $11 \quad c_{\mathrm{v}} \quad$ MacDonell, Bridle

71 NatPS234, 88

3.2,11 $c_{v}$ (BLLac-type) MacDonell, Bridle 70Nat 227, 582

$4.5 \quad c_{v}$

Lipovka 69SovAJ13, 21

5.0 a Shimmins e.a.

69AJPSup8

Shimmins, Bolton

AJPSup23 


\begin{tabular}{ll} 
Freqs & $N$ \\
\hline 5.0 & $\mathrm{a}(\mathbf{B} 2)$ \\
5.0 & OQ208
\end{tabular}

$6.6,11 \quad a$

6.6,11 BLLac

$6.6,11 \quad$ OJ 287 v

$6.6,11 \quad c_{v}$

$7.8 \quad 3 \mathrm{Cl} 20_{\mathrm{v}}$

$8.0 \quad b_{\mathrm{v}}$

8.0 b(rgal)

8.0 b(rgal)

$8.0 \quad b_{v}$

$8.0 \quad c_{v}$

$\begin{array}{ll}8.0 & \text { POO48-09 }_{\mathrm{v}} \\ \text { 8.0,11 } & \text { BLLac }_{\mathrm{v}}\end{array}$
Grueff $71 A J 76,530$

Ryle, Pooley 69ApL4,

137

Bell e.a. 71 AJ76, 524

Andrew e.a.

Andrew e.a. $71 A p L 9$,

151

Locke e.a. $69 A p J 157$,

L81

Dent 72ApJ175, L55

Brandie 72AJ77, 197

Stull 71 $A J 76,1$

Stull 71 $A J 76,970$

Stull 72AJ77, 13

Brandie, Stull

71NatPS231, 149

Macleod e.a. $71 A p L 9$,

19
69Nat 223, 598

Stull (70Nat 225, 832)

\begin{tabular}{ll} 
Freqs & $N$ \\
\hline 9.6 & $\mathrm{a}$ \\
11 & $\mathrm{a}$ \\
11 & $\mathrm{~b}_{\mathrm{v}}$ \\
18,31 & $\mathrm{c}_{\mathrm{v}}$ \\
19 & $\mathrm{c}_{\mathrm{v}}$ \\
31,85 & $\mathrm{~b}$ \\
36,130 & $3 \mathrm{C} 273_{\mathrm{v}}$ \\
69 & $\mathrm{~b}$ \\
85 & OJ287v \\
90 & $\mathrm{~b}_{\mathrm{v}}$
\end{tabular}

(iii) Flux density scales and absolute measurements

Dmitienko e.a.

71 SovAJ15, 340

Scott, Shakeshaft

$71 M N 154,19 \mathrm{P}$

Baars, Hartsuijker

72AA17, 172
Berge, Seielstad

69ApJ157, 35

Doherty e.a.

69AJ74, 827

Harvey e.a. $72 A p L 11$, 147

Hobbs, Waak

70ApJ161, 793

McCullough, Waak

69ApJ158, 849

Kellermann, Pauliny-

Toth 71ApL8, 153

Efanov e.a.

71SovAJ15, 338

Hobbs e.a. 69AJ74,

824

Kinman, Conklin

71ApL9, 147

Fogarty e.a. $71 A J 76$, 537

Conway, Munro

72MN159, 21P

Medd 72ApJ171, 41

Wrixon e.a.

72ApJ 174, 399

\section{POSITION MEASUREMENTS}

(i) Better than $\sim 1^{\prime \prime}$

\begin{tabular}{lll} 
& $N$ & Note \\
\hline b & VLBI \\
& & \\
b & VLBI \\
b & NRAO \\
b & NRAO \\
a & RRE \\
b & Cambridge \\
c & Occultation
\end{tabular}

(ii) Better than $\sim 5^{\prime \prime}$

\begin{tabular}{|c|c|}
\hline$N$ & Note \\
\hline b & Cambridge \\
\hline $\mathbf{a}$ & Cal Tech \\
\hline b & Gal \\
\hline
\end{tabular}

Cohen, Shaffer

$71 A J 76,91$

Cohen $72 A p L 12,81$

Wade 70ApJ162, 381

Wade, Miley $71 A J 76$, 101

Adgie e.a. $72 M N 159$, 233

Smith 71 NatPS232,

150

Gulkis e.a. $69 A p J 157$, 1047

Elsmore, Mackay

69MN146, 361

Fomalont, Moffet

71 AJ 76, 5

van der Kruit

71AA15, 110

\begin{tabular}{cr}
\multicolumn{1}{c}{$N$} & Note \\
\hline b & Occultation \\
APLib & Occultation \\
\hline 3 C273B & Occultation \\
\hline $3 C 279$ & Relativity \\
3 C279 & Relativity \\
3 C279 & Relativity \\
3 C279 & Relativity
\end{tabular}

Swarup e.a. $71 A p L 9$, 53

Kapahi 71NatPS234, 49

Hazard e.a. 71NatPS 233, 89

Seielstad e.a. 70PRL24, 1373

Muhleman e.a. 70PRL24, 1377

Sramek $71 A p J 167$, L55

Hill $71 M N 153,78$ van der Kruit, Katgert 72ApL11, 181

Moseley e.a.

70AJ75, 1015

Heeschen $70 A J 75$, 523 


\begin{tabular}{|c|c|c|}
\hline$N$ & Note & \\
\hline $\mathrm{a}$ & Molonglo & $\begin{array}{l}\text { Hoskins, Murdoch } \\
70 \text { AJPSup } 15\end{array}$ \\
\hline a & & Bridlee.a. $72 A J 77,405$ \\
\hline $\mathbf{a}$ & Molonglo & $\begin{array}{l}\text { Hoskins, Murdoch } \\
70 \text { AJPSup } 15\end{array}$ \\
\hline b & Molonglo & $\begin{array}{l}\text { Hunstead e.a. } \\
70 M N 149,91\end{array}$ \\
\hline $\mathbf{a}$ & Molonglo & Munro $71 A J P 24,263$ \\
\hline $\mathbf{a}$ & Molonglo & $\begin{array}{l}\text { Hunstead } 72 M N 157 \text {, } \\
367\end{array}$ \\
\hline
\end{tabular}

(iii) Less accurate

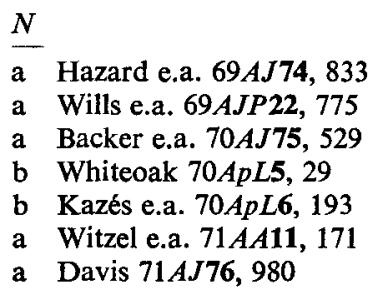

(iv) Position comparisons

\begin{tabular}{|c|c|}
\hline Note & \\
\hline Opt/radio & Shakeshaft $690 b s 89,209$ \\
\hline NRAO/RRE & Wade e.a. 70 Nat 228,146 \\
\hline Opt/NRAO & Sandage e.a. $70 A p J 162,399$ \\
\hline
\end{tabular}

\begin{tabular}{llll}
\multicolumn{1}{c}{$N$} & \multicolumn{2}{r}{ Note } & \\
\hline a & R.A. & & Clarke e.a. \\
& & 69 AJPSup 10 \\
b & QSO & & Browne, McEwan \\
& & 72 NatPS 239, 101 \\
OQ208 & & Ryle, Pooley \\
& & 69 ApLA, 137 \\
P1514-24 & & Hunstead 71 Nat 233, \\
& & 401
\end{tabular}

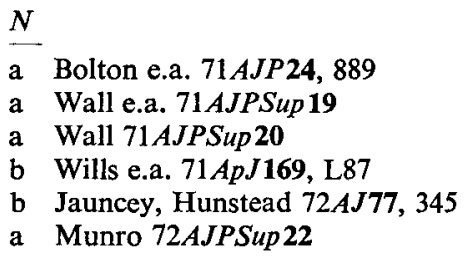

IV. BRIGHTNESS DISTRIBUTION MEASUREMENTS

(i) Pencil beam

$\begin{array}{ll}\text { c } & \text { Schilizzi 70PASA1, 337 } \\ \text { b } & \text { Kazés e.a. 70ApL6, 193 } \\ \text { b gal } & \text { Cameron 71MN152, 439 } \\ \text { a } & \text { Hunstead 72MN157, 367 } \\ \text { Centaurus A } & \text { Lockhart, Sheridan 70PASA1, 344 }\end{array}$

(ii) Interferometric: Baselines $<3 \mathrm{~km}$

\begin{tabular}{|c|c|c|}
\hline$N$ & Note & \\
\hline c & & Hogg e.a. $69 A J 74,1206$ \\
\hline a & & Ekers 69 AJPS Sup 6 \\
\hline $\mathbf{a}$ & 5C3, M31 & Pooley $69 M N 144,101$ \\
\hline b & $3 \mathrm{C}$ & Mackay $69 M N 145,31$ \\
\hline a & & $\begin{array}{l}\text { Windram, Kenderdine } \\
69 M N 146,265\end{array}$ \\
\hline c & $3 \mathrm{C}$ & Mitton $70 M N 149,101$ \\
\hline $\mathbf{a}$ & 5C4 Coma & Willson $70 M N 151,1$ \\
\hline c & $3 \mathrm{C}$ & Mitton $70 A p L 6,161$ \\
\hline b & & Fomalont $71 A J 76,513$ \\
\hline $\mathbf{a}$ & & Bridle e.a. $72 A J 77,405$ \\
\hline b & $3 \mathrm{C}$ & $\begin{array}{l}\text { Branson e.a. } 72 M N 156 \text {, } \\
377\end{array}$ \\
\hline c & Flat spec. & $\begin{array}{l}\text { Fanaroff, Blake } \\
72 M N 157,41\end{array}$ \\
\hline c & Clusters & Miley e.a. 72 Nat 237,269 \\
\hline b & Gal & Lequeux $71 A A 15,30$ \\
\hline
\end{tabular}

M33

M82

Maffei2

Maffei2

Maffei2
Terzian, Pankonin 72ApJ174, 293

Feix 72AA18, 481

Bottinelli e.a. $71 A A 12,264$

Bottinelli e.a. 71AA13, 497

Webber, Willis 71 Nat 231, 36

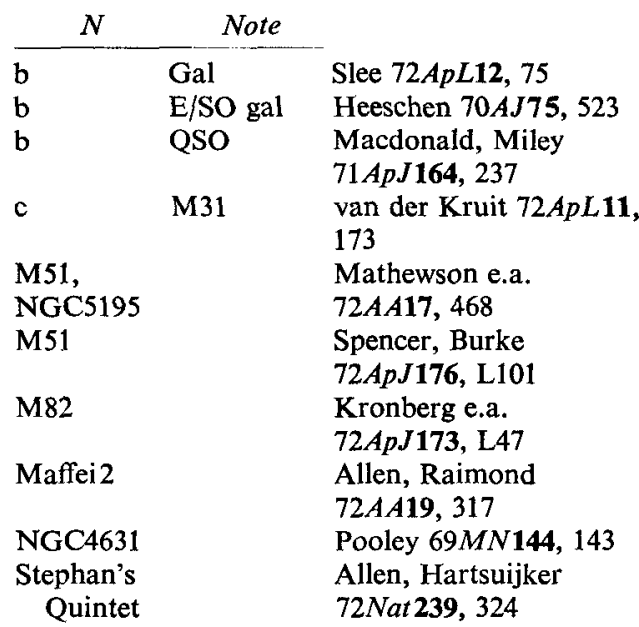




\begin{tabular}{lll}
\multicolumn{1}{c}{$N$} & Note & \\
\cline { 1 - 2 } Virgo A & Graham 70MN149, 319 \\
Virgo A & Graham 71Nat 231, 253 \\
3C9 & Clark, Miley 69ApL4, \\
3C33 & 183 \\
& Mitton 70ApL5, 207
\end{tabular}

(iii) Interferometric: Baselines $>3 \mathrm{~km}$

\begin{tabular}{|c|c|}
\hline$N$ & \\
\hline$b$ & Donaldson e.a. $69 M N 146,213$ \\
\hline & Donaldson e.a. $71 M N 152,145$ \\
\hline b & Broten e.a. $69 M N 146,313$ \\
\hline & Gubbey e.a. Nat 224, 1094 \\
\hline b & Jauncey e.a. $70 A p J 160,337$ \\
\hline & Broderick e.a. 71 SovAJ14, 627 \\
\hline & Kellermann e.a. $70 A p J 161,803$ \\
\hline & Broderick e.a. $72 A p J 172,299$ \\
\hline & Kellermann e.a. $71 A p J 169,1$ \\
\hline & Knight e.a. 71 Sc171, 52 \\
\hline & Whitney e.a. $71 S c 172,225$ \\
\hline
\end{tabular}

(iv) Occultation

$\begin{array}{ll}\text { c } & \text { Gulkis e.a. } 69 A p J 157,1047 \\ \text { c } & \text { Lang e.a. } 70 A p J 160,17 \\ \text { c } & \text { Hazard, Sutton } 71 A J 76,609 \\ \text { c } & \text { Hazard } 72 A p L 11,139\end{array}$

(v) Scintillation

$\begin{array}{llll}\text { a } & \text { Bell-Burnell 72AA16, 379 } & 3 C 48 & \text { Paniyan 69Aph5, 291 } \\ \text { APLib } & \text { Kapahi e.a. 71NatPS 234, 49 } & 3 C 273 & \text { Bell, Hewish 69ApL4, 211 } \\ \text { A.PLib } & \text { Anathakrishnan e.a. 72NatPS235, } & \text { 3C273 } & \text { Antonova e.a. 69Aph5, 283 } \\ & 167 & 3 C 273 & \text { Paniyan 70Aph6, 165 }\end{array}$

(i) Integrated linear polarization

\section{POLARIZATION MEASUREMENTS}

\begin{tabular}{ll}
$N$ & \\
\hline $\mathrm{b}$ & Cohen e.a. 71 $A p J 170,207$ \\
$\mathrm{c}_{\mathrm{v}}$ & Legg e.a. 72NatPS235, 147 \\
Cygnus A & Mitton, Ryle 69MN146, 221 \\
Cygnus A & Miley, Wade $71 A p L 8,11$ \\
M82 & Wilkinson 71MN154,1P \\
OP1934-63 & Gubbay e.a. 71 $A J 76,965$ \\
Virgo A & Cohen e.a. 69ApJ158, L83 \\
Virgo A & Miley e.a. 70ApJ159, L19/141 \\
Virgo A & Wilkinson, Peckham 72MN156, 7P \\
3C120 & Shaffer e.a. 72ApJ173, L147 \\
3C147 & Donaldson, Smith 71 $M N 151,253$
\end{tabular}

\begin{tabular}{|c|c|c|}
\hline Freqs & $N$ & \\
\hline 8.0 & $3 \mathrm{C} 279_{\mathrm{v}}$ & $\begin{array}{l}\text { Aller, Olsen } \\
71 A J 76,761\end{array}$ \\
\hline 8.0 & $\mathrm{BLLac}_{\mathrm{v}}$ & Olsen 69 Nat 224,1008 \\
\hline 9.6 & $\mathrm{~b}$ & $\begin{array}{l}\text { Berge, Seielstad } \\
69 A p J 157,35\end{array}$ \\
\hline 19 & c & $\begin{array}{l}\text { McCullough, Waak } \\
69 A p J 158,849\end{array}$ \\
\hline 31 & c & $\begin{array}{l}\text { Hobbs, Waak } \\
72 A J 77,342\end{array}$ \\
\hline 31 & c & Wardle $71 A p L 8,183$ \\
\hline
\end{tabular}

Mitton 70ApL5, 287

Riley $72 M N 157,349$ Harris 72MN158, 1 Wardle $71 A p L 8,53$ Jaffe $72 A A 20,461$

\begin{tabular}{|c|c|c|}
\hline Freqs & $N$ & \\
\hline $.41, .61$ & $\mathbf{a}$ & $\begin{array}{l}\text { Conway e.a. } \\
72 M N 157,443\end{array}$ \\
\hline .61 & a & $\begin{array}{l}\text { Kronberg, Conway } \\
70 M N 147,149\end{array}$ \\
\hline 4.2 & $\mathrm{~b}_{\mathrm{v}}$ & $\begin{array}{l}\text { Tabara e.a. } \\
72 P A S J 24,301\end{array}$ \\
\hline 5.0 & $\mathbf{a}$ & $\begin{array}{l}\text { Gardner e.a. } \\
69 A J P 22,821\end{array}$ \\
\hline $6.6,11$ & BLLac $_{v}$ & $\begin{array}{l}\text { Macleod e.a. } 71 A p L 9 \text {, } \\
19\end{array}$ \\
\hline 8.0 & $c_{\mathbf{v}}$ & Aller $70 \mathrm{Ap} J 161,1$ \\
\hline
\end{tabular}

(ii) Linear polarization distribution

$\begin{array}{ll}\text { c } & \text { Baldwin e.a. } 70 M N 150,253 \\ \text { c } & \text { Seielstad, Weiler } 71 A J 76,211 \\ \text { b } & \text { Davies, Gardner 70AJP23, 59 } \\ \text { c } & \text { Gardner, Whiteoak } 71 A J p 24, \\ & 899 \\ \text { c } & \text { Wardle } 71 A p L 8,183 \\ \text { c } & \text { Kronberg } 72 A p J 176,47 \\ \text { M51 } & \text { Mathewson e.a. } 72 A A 17,468\end{array}$

$\begin{array}{ll}\text { Cygnus A } & \text { Mitton } 71 M N 153,133 \\ \text { 3C20 } & \text { Fomalont 70ApJ160, L73 } \\ \text { 3C2721.1 } & \text { Riley 72MN157, 349 } \\ \text { 3C273 } & \text { Conway, Stannard 72NatPS239, } \\ & 22 \\ 3 C 390.3 & \text { Farris 72MN158, 1 } \\ 3 C 459 & \text { Wardle 71ApL8, 53 }\end{array}$


(iii) Circular polarization

Seielstad $69 A A 2,372$

Seaquist $70 A p L 5,111$

Gilbert, Conway 70Nat 227, 585

Seaquist 71 NatPS231, 93
Conway e.a. $71 M N 152,1 \mathrm{P}$

Biraud 72AA19, 310

Roberts e.a. 72NatPS236, 3

VI. NEUTRAL HYDROGEN OBSERVATIONS

(i) Emission: Pencil beam

\begin{tabular}{l}
\multicolumn{1}{c}{ Comment } \\
\hline $36 \mathrm{Gal}$ \\
Early type gal \\
Sb/Sc gal \\
Scd gal \\
Small gal \\
Small gal \\
Seyfert gal \\
E gal \\
Opt/21 cm \\
Opt $/ 21 \mathrm{~cm}$ \\
Opt/21 cm \\
Opt/21 cm \\
Centaurus A \\
Centaurus A \\
IC310 \\
M31 \\
M31 \\
M31 \\
M33 \\
M33
\end{tabular}
237 263 347
Bottinelli 71AA10, 437

Bottinelli e.a. 70AA6, 453

McCutcheon, Davies

$70 M N 150,337$

Rogstad, Shostak 72ApJ176, 315

Gouguenheim 69AA3, 281

Chamaraux e.a. 70AA8, 424

Allen e.a. $71 A A 10,198$

Gallagher 72AJ77, 568

Heidmann e.a. 71MRAS75, 85

Heidmann e.a. 71MRAS75, 105

Heidmann e.a. 71MRAS76, 121

Ford e.a. 71AJ76, 22

Whiteoak, Gardner 71 ApL8, 57

Roberts 70ApJ161, L9

Bottinelli e.a. $72 A A 18,121$

Davies, Gottesmann 70MN149,

Gottesmann, Davies 70MN149,

Whitehurst, Roberts $72 A p J 175$,

de Jager, Davies 71MN153, 9

Gordon 71 ApJ169, 235

\begin{tabular}{l}
\multicolumn{1}{c}{ Comment } \\
\hline M31/33 \\
M31/33 \\
M31/33 \\
M31/33 \\
M51 \\
M82 \\
M82 \\
M101 \\
M101 \\
Maffei2 \\
Maffei2 \\
NGC45 \\
NGC253 \\
NGC2403 \\
NGC3109 \\
NGC4631/4656 \\
NGC5253 \\
NGC5457 \\
NGC5457 \\
Stephan Qu. \\
Virgo A \\
IIZw40
\end{tabular}

(ii) Emission: interferometric

Comment

Techniques

Techniques

$\mathrm{Sb} / \mathrm{Sc}$ gal

\begin{tabular}{l}
\multicolumn{1}{c}{ Comment } \\
\hline NGC604 \\
M33 \\
Maffei 2
\end{tabular}

Baldwin e.a. 71MN154, 445

Wright 71 ApJ166, 455

McCutcheon, Davies

70MN150. 337

(iii) Redshifted absorption

Allen $69 A A 3,382$

Bahcall, Ekers 69ApJ157, 1055

Shuter, Gower 69Nat 223, 1046

Gottesmann, de Jager

70MRAS74, 67

Gottesmann 70MRAS74, 73

de Jager 70MRAS74, 123

Gordon 69ApL4, 47

Roberts, Warren 70AA6, 165

Guelin, Weliachew $70 A A 9,155$

Weliachew 71 PASP83, 609

Rogstad, Shostak 71AA13, 99

Rogstad, Shostak 71AA13, 108

Bottinelli e.a. 71 AA12, 264

Bottinelli e.a. $71 A A 13,497$

Lewis 72AJP25, 315

Huchtmeijer 72AA17, 207

Burns, Roberts 71ApJ166, 265

Sizikoo 71 SovAJ14, 931

Weliachew $69 A A 3,402$

Bottinelli e.a. 72AA17, 445

Guelin, Weliachew 70AA7, 141

Guelin, Weliachew 70AA9, 477

Allen 70AA7, 330

Allen 69AA3, 316

Gottesmann, Weliachew

72ApL12, 63
Wright 71 ApL7, 209

Wright e.a. 72MN155, 337

Love 72NatPS235, 53

\section{MICROWAVE BACKGROUND MEASUREMENTS}

Penzias e.a. $69 \mathrm{ApJ157,}, 49$

Alexander e.a. 69ApJ157, L163

Clark e.a. 70Nat 228,847

Pipher e.a. 71 Nat 231, 375

Heiles, Miley $70 A p J 160, \mathrm{~L} 83$

Dent $71 A p J 165,451$

Seielstad e.a. 71 $A p J 170,219$
Henry 71 Nat 231, 516

Boughn e.a. 71ApJ165, 439

Mather e.a. 71 ApJ170, L59

Thaddeus 72AnRev10, 305

VIII. MISCELLANEOUS OBSERVATIONS
$\mathrm{OH}$ in NGC253/M82
$\mathrm{H}_{2} \mathrm{O}$
Weliachew 71ApJ167, L47
Dickinson, Chaisson
71 ApJ169, 207
Source grouping
$\mathrm{X}$-ray ident.
Arp 72ApJ174, L111
Costain e.a. $72 A p J 175$, L15 
IX. OPTICAL IDENTIFICATIONS

3C Compendium

R. gal evolution

$3 \mathrm{C} /$ multiple components Van der Laan $69 A A 3,4.77$

Flux vs. spectral index Daghesmamonski 69Aph5, 297

Negative curvature

Multiple sources

Normal gal

$\mathrm{S} / \mathrm{SO}$ gal

QSO/flux density

(Bisberg 69Sov AJ13, 205

Bridle 69Nat 224, 889

Pronik 70SovA13, 747

Heeschen 70ApL6,46

Dagkesamanskii

70Nat 226, 432

Rel. Maxw. distributions Hirth 70 ApL7, 153

Interaction with background

Rowan-Robinson $70 M N 150,389$

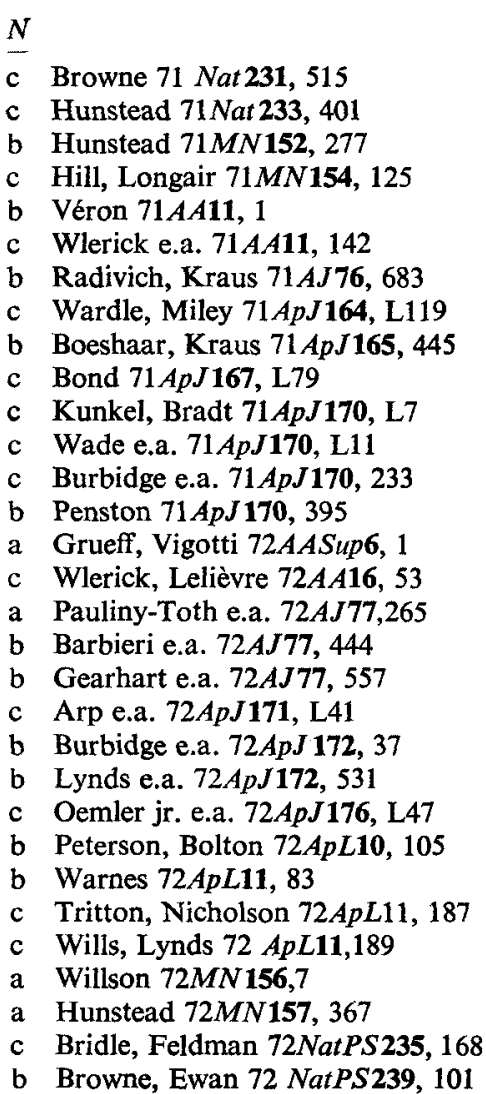

XI. INTERPRETATION OF VARIABILITY DATA/COMPACT SOURCES

3C 273 Particle accel. Simon 69ApJ158, 865 M87
Burbidge 70ApJ159, L105
Model

Radio outbursts
Kuril'chik 71ApL7, 229 Kuril'chik 71SovAJ14, 630 Kuril'chik 17 SovAJ14, 924

Non-linear spectra Braude e.a. 71 $\mathrm{ApSpSc12}$, 349 Folsom e.a. 71 NatPS 230, 199

Power vs. spectral Véron, Witzel 72AA18, index 82

Luminosity correlation Macleod,Doherty 72Nat 238, 88

Luminosity relation Bridle e.a. 72ApL11, 27 


\begin{tabular}{|c|c|c|c|}
\hline Models & $\begin{array}{l}\text { Rees, Simon 70Nat 227, } \\
1303\end{array}$ & $\begin{array}{l}\text { Changing images } \\
\text { 3C120/opt. variability }\end{array}$ & $\begin{array}{l}\text { Cavaliere } 71 S c 173,525 \\
\text { Usher } 72 A p J 172, \mathrm{~L} 25\end{array}$ \\
\hline $\begin{array}{l}\text { Intergalactic } \\
\text { scintillations }\end{array}$ & $\begin{array}{l}\text { Yoshioka } 70 P A S J 22, \\
423\end{array}$ & Model constraints & $\begin{array}{l}\text { Jones, Kellogg } \\
72 A p J 172,283\end{array}$ \\
\hline $\begin{array}{l}\text { Structure/compact } \\
\text { sources }\end{array}$ & De Young $71 A p L 9,43$ & $\begin{array}{l}\text { Multiple bursts } \\
\text { locations }\end{array}$ & Dent $72 A p J 175, \mathrm{~L} 55$ \\
\hline $\begin{array}{l}\text { Expanding source } \\
\text { model }\end{array}$ & Kogure $71 P A S J 23,449$ & $\begin{array}{l}\text { 3C279 } \\
\text { 3C279 expansion }\end{array}$ & $\begin{array}{l}\text { Dent } 72 S c 175,1105 \\
\text { Gregory } 72 N a t 239,56\end{array}$ \\
\hline
\end{tabular}

XII. INTERPRETATION OF STRUCTURE DATA

\begin{tabular}{|c|c|c|c|}
\hline Gen. discussion & Fomalont $69 A p J 157$, & Quasars & Miley $71 M N 152,477$ \\
\hline & & Radio tails/clusters & $\begin{array}{l}\text { Hill, Longair } 71 M N 154, \\
125\end{array}$ \\
\hline $3 \mathrm{C} /$ correlations & $\begin{array}{l}\text { Longair, MacDonald } \\
69 M N 145,309\end{array}$ & $3 \mathrm{C} /$ source properties & Mackay $71 M N 154,209$ \\
\hline $\begin{array}{l}\text { Min. observable diam. } \\
\text { 3C33/Ram pressure }\end{array}$ & $\begin{array}{l}\text { Harris e.a. } 70 A A 8,98 \\
\text { Mitton } 70 A p L 5,207 / 287\end{array}$ & Spectra, optical & $\begin{array}{l}\text { Kuril'chik 71SovAJ14, } \\
924\end{array}$ \\
\hline S/SO Gal & Heeschen $70 A p L 6,49$ & Cygnus $\mathrm{A} / \mathrm{ram}$ pressure & Mills $72 A p L 10,109$ \\
\hline Spiral gal & Lequeux $71 A A 15,42$ & Radio trails/clusters & Miley e.a. 72 Nat 237,269 \\
\hline $\begin{array}{l}\text { Double sources/ram } \\
\text { pressure }\end{array}$ & Wardle 71 ApL8, 221 & $\begin{array}{l}\text { Double sorces/ram } \\
\text { pressure }\end{array}$ & $\begin{array}{l}\text { Kuril'chik } 72 \text { SovAJ15, } \\
542\end{array}$ \\
\hline
\end{tabular}

Orientations/E gal Mackay 71MN151, 421

XIII. INTERPRETATION OF POLARIZATION DATA

$\begin{array}{ll}\text { Quasars/spectral index } & \text { Gilbert e.a.69Nat 223, } \\ & 1252 \\ 8 \mathrm{GHz} & \text { Aller } 70 \mathrm{ApJ161}, 19\end{array}$

Expandingsource model Aller 70Nat 225, 440

Vs. redshift Conway, Gilbert

Vs. redshift

70 Nat 226, 332

Gardner, Whiteoak

70Nat 227, 585
Magn. field scales

Linear vs. circular

Rotation measures

Rotation measures

3C Compendium

Faraday depolarization Strom 72NatPS239, 19
Perola $71 A A 14,337$

Melrose $71 A p L 8,227$

Reinhardt 72AA19, 104

Mitton, Reinhardt

72AA20, 337

Strom 72NatPS239, 19

\section{REDSHIFT DEPENDENT PROPERTIES OF SOURCES}

\begin{tabular}{|c|c|c|c|}
\hline $\begin{array}{l}\text { Ang. size/weak } \\
\text { sources }\end{array}$ & $\begin{array}{l}\text { Longair, Pooley } \\
69 M N 145,121\end{array}$ & Polarization & $\begin{array}{l}\text { Gardner, Whiteoak } \\
70 \text { Nat 227, } 585\end{array}$ \\
\hline $\begin{array}{l}\text { Ang. size/double } \\
\text { sources }\end{array}$ & Legg 70 Nat 226, 65 & $\begin{array}{l}\text { Ang. size } \\
\text { Opt. ys. HI/anomalous }\end{array}$ & $\begin{array}{l}\text { Miley } 71 M N 152,477 \\
\text { Lewis } 71 N a t P S 230\end{array}$ \\
\hline Polarization & Conway, Gilbert & redshifts & \\
\hline & 70 Nat 226, 332 & Criticism of Lewis & Arp 71 NatPS231, 103 \\
\hline Flux/radio galaxies & Hoyle, Burbidge & Faraday rotation & Arp 71 Nat 232, 463 \\
\hline & 70 Nat 227, 359 & Depolarization & $\begin{array}{l}\text { Kronberg e.a. } \\
72 M N 156,275\end{array}$ \\
\hline \multicolumn{4}{|c|}{ XV. MISCELLANEOUS CORRELATIONS } \\
\hline Source pairs & $\begin{array}{l}\text { Hinder, Branson } \\
69 \text { Obs89, } 178\end{array}$ & $\begin{array}{l}\text { Source grouping } \\
\text { Seyferts/radio vs. }\end{array}$ & $\begin{array}{l}\text { Arp 72ApJ174, L111 } \\
\text { Rieke, Low 72ApJ176, }\end{array}$ \\
\hline $\begin{array}{l}\text { Small source/opt. } \\
\text { peculiar gal. nuclei }\end{array}$ & Tifft $70 A p L 7,7$ & $\begin{array}{l}\text { infrared } \\
\text { Source orientation/ }\end{array}$ & $\begin{array}{l}\text { L95 } \\
\text { Willson } 72 M N 155,275\end{array}$ \\
\hline Source randomness/ & Wills 71NatPS234, 168 & grouping & \\
\hline
\end{tabular}

XVI. INTERPRETATION OF MICROWAVE BACKGROUND DATA

Hazard, Salpeter 69ApJ157, L87

Dautcort 69MN144, 255

Longair, Sunyaev 69Nat 223, 719

Sunyaev, Zeldovich 69 Nat 223, 721

Brecher, Blumenthal 70ApL6, 169
Sunyaev, Zeldovich 70ComA2, 66

Rowan-Robinson $70 M N 150,389$

Rasband $71 \mathrm{ApJ170,1}$

Caroff, Petrosian 71Nat 231, 378

Fanaroff, Longair 72MN159, 119 
(i) Radiation Theory

Collective

Bremstrahlung

Papadopoulos, Lerche

69ApJ 158, 981

Relativistic Streaming Noerdlinger 69ApL4, 233

Synchotron

Developments

Limitations/small pitch angles

Turbulent plasma

69AnRev7, 375

O'Dell, Sartori

$70 A p J 161$, L63

Colgate e.a. $70 \mathrm{ApJ} 162$,

\section{THEORY OF RADIO SOURCES} 649

(ii) Effects of Compton Scattering

\begin{tabular}{|c|c|}
\hline Secondary electrons & Perola $69 A A 3,481$ \\
\hline Electron acceleration & Burke, Layzer \\
\hline & $69 A p J 157,1169$ \\
\hline Low freq. cut-off & O'Dell, Sartori \\
\hline & $70 A p J 162, \mathrm{~L} 37$ \\
\hline Low freq. spectrum & Sunyaev $70 A p L 7,19$ \\
\hline Radiative heating & Levitch, Sunyaev \\
\hline (iii) Source models & \\
\hline QSO's/pulsars & Morrison $69 A p J 157, \mathrm{~L} 73$ \\
\hline $\begin{array}{l}\text { Rotating collapsed } \\
\text { objects }\end{array}$ & $\begin{array}{l}\text { Cavaliere e.a. } 69 A p L 4 \text {, } \\
103\end{array}$ \\
\hline $\begin{array}{l}\text { Galactic nuclei-old } \\
\text { quasars }\end{array}$ & $\begin{array}{l}\text { Lynden-Bell 69 Nat 223, } \\
690\end{array}$ \\
\hline Formation of jets & $\begin{array}{l}\text { Bisnovatyi-Kogan e. a. } \\
69 \text { Sov } A J 13,369\end{array}$ \\
\hline Patching model & $\begin{array}{l}\text { Melik-Alaverdion } \\
70 \text { Aph6, } 341\end{array}$ \\
\hline Electrodynamic model & $\begin{array}{l}\text { Piddington } 70 M N 148 \text {, } \\
131\end{array}$ \\
\hline Pulsar in QSO's & Sturrock $71 A p J 170,85$ \\
\hline $\begin{array}{l}\text { Multiple explosions/ } \\
\text { heating }\end{array}$ & $\begin{array}{l}\text { Christiansen } 71 A p L 7 \text {, } \\
233\end{array}$ \\
\hline Magn. field generation & Bisnovatyi-Kogan, \\
\hline
\end{tabular}

(iv) Magnetohydrodynamics/Source Confinement

Ram pressure model Christiansen $69 M N 145$, 327

Particle diffusion

Ram pressure model

3 C33

Hydromagnetic bubbles/double sources

(i) Data

Frq

.41 4C Identifications Munro 71 AJP24, 617

1.4 Ohio

Harris, Kraus 70 Nat 227, 785

Ohio flux error

Jauncey, Niell

71 Nat PS 229, 223

Ohio flux error

Harris, Kraus

71 NatPS 230, 140

Anisotropic emission

Small pitch angles

Plasma approximations

Circ. polarization

Compton/spectrum depression

Circ. polarization

Compact sources

Spectrum bending

Low freq. spectrum

Compact sources

Superlight sources

Synchro-compton

Synchro-compton

Outbursts

Synchro-compton

Energy source/ structure

Gal. \& QSO activity

Nuclei ejection

Synchro-compton

Variability

Superlight expansion

Dynamics/extended sources

Ram pressure vs. observations

Cygnus A

Ram pressure instabilities

Gas in clusters
Kuril'chik 70SovAJ14, 21

Melrose 71 ApL8, 35

Wild, Hill 71 AJP24, 43

Pacholczyk, Swihart

71MN153, 3P

Getmansev, Tokarev

72ApL12, 57

Rosenberg 72AA19, 66
$70 \mathrm{ApL7}, 69$

Walmsley $71 \mathrm{ApL8}, 27$

Jaffe, Treves $71 A p L 9,39$

Sunyaev 71 Sov $A J 15,190$

Olster, Alschuler

71 ApSpSc10, 431

Ginzburg 72ComA4, 41

Vainstein $71 A p L 8,151$

Rees 71Nat 229, 312

Jennison 71 NatPS231, 88

Shklovsky 71 Sov $A J 14$,

594

Blandford 72AA20, 135

Daltabuit, Cox

72ApJ 173, L13

Sturrock, Barnes

72ApJ 176, 31

Shklovsky $72 A p L 10,5$

Blandford, Rees

72ApL10, 77

Kuril'chik $72 A p L 10,115$

Richter 72NatPS 237, 71

De Young $71 A p J 167$, 541

Wardle $71 A p L 8,221$

Mills 72ApL10, 109

Blake $72 M N 156,67$

Miley e.a. $72 N a t 237,269$

XVIII. SOURCE COUNTS AND RELATED TOPICS

Frq

Ohio extension

1.4 Spectral indices

1.4

5.0

8.0 Flat spectra
Kraus 72NatPS236, 5

Willson $72 M N 155,385$

Bridle e.a. 72NatPS235, 123

Kellermann e.a.

71ApJ170, L1

Brandie 70Nat 225, 352 
(ii) Interpretation and luminosity functions

\begin{tabular}{|c|c|c|c|}
\hline Counts/review & $\begin{array}{l}\text { Brecher e.a. } 71 \text { ComAIII, } \\
99\end{array}$ & Evolution function & $\begin{array}{l}70 M N 147,139 \\
\text { Ringenberg, McVittie }\end{array}$ \\
\hline Counts/review & Longair, Rees & & $70 M N 149,341$ \\
\hline & 72 ComAIV, 79 & Counts/interpretation & Rowan-Robinson \\
\hline uminosity-volume test & Schmidt $70 A p J 162,371$ & & $70 M N 149,365$ \\
\hline uminosity-volume test & $\begin{array}{l}\text { Longair, Scheuer } \\
70 M N 151,45\end{array}$ & Quasar evolution & $\begin{array}{l}\text { Cavalier e.a. } 71 A p J 170 \text {, } \\
223\end{array}$ \\
\hline Luminosity-volume test & $\begin{array}{l}\text { Rees, Schmidt71MN154, } \\
1\end{array}$ & Rgal evolution & $\begin{array}{l}\text { Rowan-Robinson } \\
71 \text { Nat } 229,388\end{array}$ \\
\hline uminosity-volume test & Caswell, Weyman & Critic & Mackay 71 Nat 233,402 \\
\hline Luminosity-volume test & $\begin{array}{l}72 M N 156,19 \mathrm{P} \\
\text { Lynds, Petrosian }\end{array}$ & f Mackay & $\begin{array}{l}\text { Rowan-Robinson } \\
71 \text { Nat 233, } 403\end{array}$ \\
\hline & $72 A p J 175,591$ & & Davidson 70 Nat 227,357 \\
\hline tionar & Arakelian $69 A p h 5,461$ & & \\
\hline $\begin{array}{r}\text { qso's } \\
\text { Evolutionary effects/ } \\
\text { qso's }\end{array}$ & Arakelian $69 A p h 5,603$ & $\begin{array}{l}\text { Luminosity vs. density } \\
\text { evolution } \\
\text { Secular evolution }\end{array}$ & $\begin{array}{l}\text { Davidson e.a. } 71 \text { AJP24, } \\
403 \\
\text { Davidson } 71 M N 154,339\end{array}$ \\
\hline $\begin{array}{r}\text { Sources-background/ } \\
\text { s.state }\end{array}$ & $\begin{array}{l}\text { Hazard, Salpeter } \\
69 A p J 157, \mathrm{~L} 87\end{array}$ & $\begin{array}{l}\text { Observational selection/ } \\
\text { 3C qso's }\end{array}$ & $\begin{array}{l}\text { Lynden-Bell } 71 M N 155 \text {, } \\
95\end{array}$ \\
\hline $\begin{array}{l}\text { Source evolution/ } \\
\text { background }\end{array}$ & Longair $70 M N 150,155$ & $\begin{array}{l}\text { Bright galaxies } \\
\text { Rgal luminosity }\end{array}$ & $\begin{array}{l}\text { Cameron } 71 M N 152,429 \\
\text { Merkelijn } 71 A A 15,11\end{array}$ \\
\hline $\begin{array}{l}\text { Sources/interaction } \\
\text { with background }\end{array}$ & $\begin{array}{l}\text { Rowan-Robinson } \\
70 M N 150,389\end{array}$ & $\begin{array}{l}\text { function } \\
\text { QSO/opt and radio }\end{array}$ & $71 \mathrm{Aph} 7,457$ \\
\hline Fitting count data & $\begin{array}{l}\text { Crawford e.a. } \\
70 A p J 162,405\end{array}$ & $\begin{array}{l}\text { QSO luminosity } \\
\text { evolution }\end{array}$ & $\begin{array}{l}\text { Golden } 71 \text { NatPS234, } \\
103\end{array}$ \\
\hline Luminosity function & $\begin{array}{l}\text { Windram, Kenderdine } \\
69 M N 146,265\end{array}$ & $\begin{array}{l}\text { Density/luminosity } \\
\text { function }\end{array}$ & $\begin{array}{l}\text { Edwards } 71 \text { NatPS232, } \\
59\end{array}$ \\
\hline $\begin{array}{r}\text { Luminosity evolution/ } \\
\text { qso's }\end{array}$ & Arakelian 70 Nat 225, 358 & $\begin{array}{l}\text { Reply to Edwards } \\
\text { Rgal, QSO evolution }\end{array}$ & $\begin{array}{l}\text { Longair 71NatPS232, } 59 \\
\text { Bahcall } 72 A p J 172,265\end{array}$ \\
\hline $\begin{array}{l}\text { Luminosity function/, } \\
\text { qso's }\end{array}$ & Arakelian $70 A p h 6,531$ & $\begin{array}{l}\text { QSO evolution } \\
\text { Rgal evolution }\end{array}$ & $\begin{array}{l}\text { Schmidt 72ApJ176, } 273 \\
\text { Schmidt 72ApJ176, } 289\end{array}$ \\
\hline $\begin{array}{l}\text { Luminosity function/ } \\
\text { Evolution/large z }\end{array}$ & Alaverdian $70 \mathrm{Aph6}, 54$ & $\begin{array}{l}\text { Source count interpret. } \\
\text { Evolution/large sources }\end{array}$ & $\begin{array}{l}\text { Schmidt } 72 A p J 176,303 \\
\text { Fanaroff, Longair } \\
72 M N 159,119\end{array}$ \\
\hline
\end{tabular}

XIX. MISCELLANEOUS RADIO-COSMOLOGY

\begin{tabular}{|c|c|c|c|}
\hline Antipodal images/ & Andretch, Dehnen & & $72 A A 17,432$ \\
\hline $\begin{array}{c}\text { cosmology } \\
\text { Ghost images/ }\end{array}$ & $\begin{array}{l}69 A A 3,252 \\
\text { Petrosian. Ekers }\end{array}$ & $\begin{array}{l}\text { Grav. deflection/ } \\
\text { relativity }\end{array}$ & $\begin{array}{l}\text { Seielstad e.a. } 70 P R L 24, \\
1373\end{array}$ \\
\hline $\begin{array}{l}\text { Ghost images/ } \\
\text { statistics }\end{array}$ & $\begin{array}{l}\text { Petrosian, Ekers } \\
\text { 69NatPS 224, } 484\end{array}$ & $\begin{array}{l}\text { relativity } \\
\text { Grav. deflection/ }\end{array}$ & $\begin{array}{l}1373 \\
\text { Muhleman e.a. }\end{array}$ \\
\hline $\begin{array}{l}\text { Metagalactic field/ } \\
\text { Faraday rotation }\end{array}$ & Kawabàta e.a. & relativity & 70PRL24, 1377 \\
\hline $\begin{array}{l}\text { Faraday rotation } \\
\text { Intergal. magn. field }\end{array}$ & $\begin{array}{l}\text { 69PRASJ21, } 293 \\
\text { Brecher, Blumenthal }\end{array}$ & $\begin{array}{l}\text { Grav. deflection/ } \\
\text { relativity }\end{array}$ & Sramek $71 A p J 167$, L55 \\
\hline rotation/ & $\begin{array}{l}70 A p L 6,169 \\
\text { Burman } 72 P A S J 24,291\end{array}$ & $\begin{array}{l}\text { Grav. deflection/ } \\
\text { relativity }\end{array}$ & Hill $71 M N 153,78$ \\
\hline & & Local/cosmological & Rov \\
\hline Cluster magn. field & Perola, Reinhardt & QSO's & 72 Nat 236, 112 \\
\hline
\end{tabular}

XX. REVIEW PAPERS ETC.

(i) Compendia of data

$\begin{array}{llll}\text { Parkes catalogue } & \text { CSIRO staff 69AJPSup7 } & \text { QSO's } & \text { De Veny e.a. } \\ \text { 3C spectra } & \text { Kellermann e.a. } & & \text { 71PASP83, 611 } \\ & \text { 69ApJ157, 1 } & \text { 3C polarizations } & \text { Mitton, 72MN155, 373 } \\ \text { Sources/master list } & \text { Dixon 70ApJSup20, 1 } & & \end{array}$


(ii) Reviews

\begin{tabular}{|c|c|}
\hline Nuclei of gal. & $\begin{array}{l}\text { Semaine d'Etude } 71 \text {, } \\
\text { Vatican }\end{array}$ \\
\hline Gal/QSO-IAUSymp. 44 & Evans 70Reidel \\
\hline Short $\mu$ wave obs. & Foster $69 Q J 10,206$ \\
\hline History/M33 & Gordon $69 Q J 10,293$ \\
\hline $\begin{array}{l}\text { Synchotron develop- } \\
\text { ments }\end{array}$ & $\begin{array}{l}\text { Ginzburg, Syrovatskii } \\
69 \text { AnRev7, } 375\end{array}$ \\
\hline QSO's & Schmidt $69 \operatorname{AnRev7,527}$ \\
\hline $\begin{array}{l}\text { High resolution } \\
\text { observations }\end{array}$ & Cohen 69 AnRev7, 619 \\
\hline QSO's & Burbidges $69 N a t 224,21$ \\
\hline Infra-red $/ \mu$ wave & $\begin{array}{l}\text { Feldman e.a. } 69 \text { Nat 224, } \\
752\end{array}$ \\
\hline Gal nuclei & Burbidge $70 A n \operatorname{Rev8}, 369$ \\
\hline
\end{tabular}

$\begin{array}{ll}\text { Radio Astrophysics } & \text { Pacholczyk 70Freeman } \\ \text { Compact Gal/QSO's } & \text { Zwicky 70AdvAA7, 228 } \\ \text { Energy problems } & \text { Ryle 70QJ11, 429 } \\ \text { Unsolved problems } & \text { Bahcall } 71 A J 76,283 \\ \text { Cosmical constant } & \text { McCrea } 71 Q J 12,140 \\ \text { Magn. fields } & \text { Cowling } 71 Q J 12,348 \\ \text { Magnetohydrodynamic } & \text { Tayler 71QJ12, 352 } \\ \quad \text { stability } & \\ \text { Source counts/ } & \text { Brecher e.a. 71ComA3, } \\ \quad \text { theories } & 99 \\ \text { Nature of redshifts } & \text { Arp 71Sci174, 1189 } \\ \text { Cosmic ray electrons } & \text { van de Hulst 72QJ13, 10 } \\ \text { Source counts/ } & \text { Longair, Rees 72ComA4, } \\ \quad \text { interpretation } & 79\end{array}$

\section{RADIO ASTRONOMY INSTRUMENTS}

\section{R. Wielebinski}

\section{Radio telescopes}

The last three years saw the completion of a number of large radio telescopes. New filled aperture telescopes and a number of array and synthesis array telescopes will be described. A very useful discussion of all aspects of radio telescopes can be found in a book by Christiansen and Högbom(1).

\section{Filled apertures}

A survey of filled aperture radio telescopes was made by Findlay (2). Of the fully steerable paraboloidal reflectors completed, the largest is the 100 -m radio telescope $(3,4)$ of the Max-PlanckInstitut für Radioastronomie. One of the features of the telescope is the use of 'homologous' design. The elastic structure supporting the paraboloidal surface deforms into a series of paraboloids as the telescope is tipped from zenith to the horizon. The changing focus position can easily be followed with small movements of the feed. The MPIfR $100-\mathrm{m}$ telescope operates successfully at $2.8 \mathrm{~cm}$ wavelength indicating the usefulness of the design principle. A number of smaller reflectors usable down to $\mathrm{mm}$-wavelengths have also been completed. Data on high resolution mm-reflector antennas has been summarized by Cogdell et al. (5). Details of the 22-m dish in Crimea capable of operation down to $1 \mathrm{~mm}$ wavelength can be found separately (6). Studies of larger mm-wave telescopes have also been completed in view of the rapid development of $\mathrm{mm}$ wavelength spectroscopy, and construction of such antennas can be expected in the future.

\section{Array telescopes}

Numerous unfilled aperture telescopes have come into operation during the last three years. The largest of these is the 5-km synthesis array at Cambridge (7) which will have a resolution of 2 " at 6-cm wavelength. The Westerbork synthesis array (8) is now fully operational at $6 \mathrm{~cm}, 21 \mathrm{~cm}$ and $49 \mathrm{~cm}$. The Fleurs telescope, the first synthesis telescope in the southern sky was commissioned in 1972 (9). One of its features is the simultaneous correlation for all interferometer pairs allowing a synthesis to be complete after $12 \mathrm{hr}$ of observing. Another synthesis instrument is Stanford's high resolution radio interferometer (10). One of the more novel instruments to become operational in this period is the Ooty array (11). This telescope uses the natural geographical position (latitude $11^{\circ}$ ) to give a telescope which requires to be steered only in one axis. Numerous lunar occultations have been observed.

The advent of space radio astronomy was announced in the last report. Improvements allow observations to be carried out in the frequency range from some $100 \mathrm{kHz}$ to $10 \mathrm{MHz}(12)$. 
Numerous other instruments, smaller than those reported here have become operational in the past three years. They are too numerous to be included here.

\section{Antenna feeds}

The efficient use of any reflector radio telescope depends on the use of good feeds. This is particularly true for large paraboloidal or spherical reflectors. The field distributions in the focal region have been investigated $(13,14)$ and can readily be computed. Then a feed must be designed which on immersion in these fields matches the energy distribution without altering the amplitude and phase relations. One of the most successful methods is to use a hybrid mode feed (15). For deep paraboloidal dishes feeds based on a scalar horn design have been developed (16). Another approach has been the use of coaxial waveguides (17) with exact control of phase and amplitude. Efficiencies of $80 \%$ are being approached in practice depending on the complexity of the feed.

\section{Receivers}

There has been a steady development of receiver front-ends and an explosive development in the field of digital hardware.

\section{Front-ends}

There has been an extension in the frequency range, reduction in the receiver noise and increase in the bandwidth of parametric amplifiers. Uncooled parametric amplifiers have been made to operate at $46 \mathrm{GHz}(\mathbf{1 8})$ using waveguide mounted varactor diodes. Cooled parametric amplifier performance has been steadily improved by the use of higher pump frequencies, better diodes and improvements in design of cooled circulators. Low-loss cooled circulators (19) are a critical component and can now be commercially produced by a number of manufacturers, resulting in low receiver noise temperatures. The MASER as a front-end receiver is being reinvestigated particularly for high frequencies. The maser has a somewhat lower nôise temperature but only a narrow bandwidth, but this is no disadvantage for most spectral line observations. For mm-wavelengths receivers from Bell Telephone Laboratories (20) and NRAO using specially developed Schottky-barrier diodes mounted in a waveguide appear to be the only practically successful devices. However, this field of receiver technology is in rapid development and hopefully, a significant breakthrough may be at hand.

\section{Back-ends}

In the last three years rapid development took place in the field of receiver systems, particularly in digital hardware. Each large observatory now has a large autocorrelation spectrograph. Descriptions can best be found in internal reports, and the NRAO Electronics Series can be particularly recommended for reference. With the decreasing cost of digital chips spectrographs with 1000 or more channels and with multi-bit operation have become feasible. Analog to digital converters can now be made cheaply and as a consequence are widely used in receiving equipment. A small computer can then transfer partly reduced data onto a magnetic tape. The discovery of pulsars has brought about the development of many special purpose receiving systems, particularly for de-dispersion applications. One other significant development has been the NRAO Mark II VLB terminal (21) which now has been delivered to some 12 users all over the world.

Unfortunately many technical developments are never completely documented or published and duplication of designs often occurs.

\section{BIBLIOGRAPHY}

1. Christiansen, W. N., Högbom, J. A. 1969 , Radiotelescopes, Cambridge University Press.

2. Findlay, J. W. 1971, Ann. Rev. Astron. Astrophys. 9, 271. 
3. Hachenberg, O. 1970, Sky Telesc. 40, 338.

4. Wielebinski, R. 1971, Naturwissenschaften 58, 109.

5. Cogdell, J. R., et al. 1970, I.E.E.E. Trans. A-P 18, 515.

6. Viestavkin., et al. 1971, Izvestia Crimean Akademie, 43, 26.

7. Ryle, M. 1972, Nature, $239,435$.

8. Oort, J. H. 1971, 'De Synthese Radiotelescoop in Westerbork', Z.W.O. Jaarboek 1970, s'Gravenhage.

9. Christiansen, W. N. 1972, 'The Fleurs Synthesis Radiotelescope, University of Sydney.

10. Bracewell, R. N., Colvin, R. S., Price, K. M., Thompson, A. R. 1971, Sky Telesc. 42, 2.

11. Swarup, G., et al. 1971 , Nature Phys. Sci., 230, 185.

12. Weber, R. R., Alexander, J. K., Stone, R. G. 1971, Radio Sci. 6, 1085.

13. Thomas, McA. B. 1970 , I.E.E.E. Trans., AP-18, 404.

14. Rumsey, V. H. 1970, I.E.E.E. Trans., AP-18, 343.

15. Thomas, McA. B. 1970 , Electronics Letters, 6, No. 15.

16. Wohileben, R., Mattes, H., Lochner, O. $\quad 1972$, Electronics Letters, 8, 474.

17. Koch, F. G., Scheffer, H., Thielen, H. 1972, Nachrichtentechn. Fachberichte, 45, 70.

18. Edrich, J. 1971, Proc. I.E.E.E., 59, 1125.

19. Edrich, J., West, R. G. 1970, I.E.E.E., MTT-18, 743.

20. Penzias, A. A., Burrus, C. A. 1972 , Ann. Rev. Astron. Astrophys., 10 (in press).

21. Clark, B. G., Weimer, R., Weinreb, S. 1972, NRAO Electronics Division Internal Report, 118.

D. S. HEESCHEN

President of the Commission 